\title{
Long-Term Treatment With Cisapride and Antibiotics in Liver Cirrhosis: Effect on Small Intestinal Motility, Bacterial Overgrowth, and Liver Function
}

\author{
Ana Maria Madrid, M.D., Carmen Hurtado, Ph.D., Mauricio Venegas, Ph.D., Francisco Cumsille, Dr. P.H., \\ and Carlos Defilippi, M.D. \\ Gastrointestinal Section, University Hospital, University Of Chile, Santiago, Chile
}

OBJECTIVES: Altered small-bowel motility, lengthening of the orocecal transit time, and small-intestinal bacterial overgrowth have been described in patients with liver cirrhosis. These changes might be related to the progressive course and poor prognosis of the disease. We investigated the effect of a long-term treatment with cisapride and an antibiotic regimen on small-intestinal motor activity, orocecal transit time, bacterial overgrowth, and some parameters of liver function.

METHODS: Thirty-four patients with liver cirrhosis of different etiology entered in the study. They were randomly allocated to receive cisapride (12), an alternating regimen of norfloxacin and neomycin (12), or placebo (10) during a period of 6 months. At entry and at 3 and 6 months, a stationary small-intestinal manometry was performed, and orocecal transit time and small-intestinal bacterial overgrowth were also investigated using the $\mathrm{H}_{2}$ breath test. Liver function was estimated with clinical and laboratory measurements (Child-Pugh score).

RESULTS: After 6 months, both cisapride and antibiotics significantly improved fasting cyclic activity, reduced the duration of orocecal transit time, and decreased small-intestinal bacterial overgrowth. Cisapride administration was followed also by an increase in the amplitude of contractions. No statistically significant variations in these parameters were observed with placebo. An improvement of liver function was observed at 3 and 6 months with both cisapride and antibiotics.

CONCLUSIONS: Long-term treatment with cisapride or antibiotics reversed altered small-intestinal motility and bacterial overgrowth in patients with liver cirrhosis. These findings suggest a possible role for prokinetics and antibiotics as a modality of treatment in selected cases of decompensated cirrhosis. (Am J Gastroenterol 2001;96:1251-1255. (C) 2001 by Am. Coll. of Gastroenterology)

\section{INTRODUCTION}

Altered small-bowel motility in patients with liver cirrhosis was described by Chesta et al. (1) and confirmed by other studies (2). Abnormalities were observed during the fasting period and were characterized by alterations in the migrating motor complex (MMC), ranging from increased duration of phase II of the MMC to the complete disappearance of the cyclic activity. An increase in the prevalence of clustered contractions was also described. Additional studies showed that the appearance and intensity of these motor abnormalities were related to the severity of liver dysfunction (2) and that alterations of motility observed in patients with liver cirrhosis were completely reversed by liver transplantation (3).

A prolonged orocecal transit time (OCTT) has also been observed in patients with liver cirrhosis $(4,5)$. When gastric emptying was simultaneously analyzed, the pylorus-caecum component of OCTT played the main role (4). Nevertheless, the relationship between altered small-intestinal motility and delayed small-intestinal transit time in these patients has not been investigated.

Small-intestinal bacterial overgrowth (SIBO) has also been described in patients with liver cirrhosis $(6,7)$. The appearance and the frequency of this finding was related to the severity of the liver disease. Also, an increased incidence of spontaneous bacterial peritonitis was observed in patients with SIBO (8).

Therefore, the available data suggests that when liver function is progressively impaired, an increased intensity of altered motility and SIBO is observed.

At present, the relationship between small-intestinal motor disorders and SIBO is not completely established. It is possible that liver disease and associated autonomic disorders leading to altered small-intestinal motility may result in SIBO. Absence or gross disorders of the MMC were associated with SIBO also in patients without liver disease, and motor abnormalities remained unchanged after treatment with antibiotics (9). Altered small-intestinal motility was not reversed in patients with liver cirrhosis after a short treatment with tetracycline (1). On the other hand, SIBO might be present in cirrhosis or a consequence of hypochlorhydria or immunological defects, and therefore the possibility that bacterial overgrowth might induce changes of motility cannot be completely excluded.

In a preliminary study, we demonstrated that intravenous 
cisapride was followed by the appearance of a phase III of the MMC in cirrhotics in which cyclic activity was absent (10). Therefore, the aims of the present study were as follows: 1) to investigate the effect of oral cisapride administered over a long period of time on the cyclic motor activity of patients with liver cirrhosis and, to also analyze the effect of this prokinetic drug on SIBO, OCTT, and the severity of liver disease; 2) to analyze the effect of a longterm antibiotic treatment on the same parameters.

\section{MATERIALS AND METHODS}

\section{Patients}

The study involved 34 patients with liver cirrhosis (15 men and 19 women; mean age, $57.6 \mathrm{yr}$; range, 32-69 yr).

The etiology of cirrhosis was of alcoholic origin in 18 subjects, related to hepatitis $\mathrm{C}$ virus in nine, and from other etiologies in the remaining subjects. The diagnosis in each case was confirmed by appropriate investigations, including laboratory investigations, liver biopsy, and endoscopy. According to the Child-Pugh classification, six patients were in class A, 20 were in class B, and eight were in class C.

Patients excluded were those with diabetes mellitus, cardiovascular disorders, altered EKG, marked serum electrolyte disturbances, spontaneous bacterial peritonitis, previous abdominal surgery and renal failure, or those who had been treated with lactulose, antibiotics, or prokinetic drugs during the past 30 days. All patients signed an informed consent. This study was approved by the ethical committee of the University Hospital of the University of Chile.

\section{Motility Studies}

Duodenal motor activity was studied by means of a multilumen-perfused catheter assembly, which incorporated four polyvinyl tubes (internal diameter, $0.9 \mathrm{~mm}$ ) glued together with tetrahydrofuran and with side holes spaced $3 \mathrm{~cm}$ from each other. They were continuously infused with bubblefree distilled water with a pneumohydraulic capillary infusion system (Arndorfer Medical Specialities, Greendale, WI) at a rate of $0.4 \mathrm{~mL} / \mathrm{min}$. The manometric catheters were attached to external TP-400t pressure transducers and connected to a Nihon Kohden polygraph (Nihon Kohden, Tokyo, Japan).

The output of the amplifiers was in turn connected to an analog-digital converter in a personal computer, and signals were analyzed by a computer program developed in our laboratory.

After an overnight fast, the catheter assembly was passed by mouth to the stomach and was advanced under fluoroscopic control until the tip of the assembly had reached the angle of Treitz, the recording sites being located in the third and fourth part of the duodenum. Studies were performed in the fasting state only.

\section{Analysis of Data}

MOTOR ACTIVITY. The different phases of the MMC and clustered contractions were identified by direct visual inspection of the chart-recording paper using the following criteria: phase I was characterized by the complete absence of contractions; phase II consisted of irregular phasic contractions; these culminated in a burst of rhythmic phasic contractions at a frequency of $12 \mathrm{cpm}$ (phase III). To define MMC activity, the presence of at least one phase III preceded by phase II and followed by phase I was required. Clustered contractions were defined as a sequence of three to 10 rhythmic contractions preceded and followed by a quiescent period of 1 to $5 \mathrm{~min}$ duration. According to previous studies in the fasting period (11), more than six clusters per hour occurring at all four sites was considered abnormal.

The frequency and amplitude of contractions were calculated by means of the automatic system.

$\mathrm{H}_{2}$ BREATH TEST. OCTT and SIBO were estimated by means of the $\mathrm{H}_{2}$ breath test. Hydrogen concentration expressed as parts per million (ppm) in end expiratory air was measured by means of an automatic analyzer (Lactoscreen Hoek, Loos Schiedom, Holland). Patients were instructed to eat a low-carbohydrate food at 8 PM the day before the test. Hydrogen breath test was performed at $8 \mathrm{AM}$, preceded by a mouthwash with $0.1 \%$ hexetidine. Two or three basal $\mathrm{H}_{2}$ measurements were performed, and then subjects received $12 \mathrm{~g}$ of lactulose (Duphalac, Solvay Pharmaceutical B.V., Holland) dissolved in $300 \mathrm{~mL}$ of distilled water, after which measurements of $\mathrm{H}_{2}$ concentration were performed every 10 min over a period of $180 \mathrm{~min}$. The sum of the following criteria was included to establish that a patient presented with SIBO: basal values $>20 \mathrm{ppm}$ and $\mathrm{H}_{2}$ concentrations $\geq 100 \mathrm{ppm}$ in the first $60 \mathrm{~min}$. OCTT was considered from lactulose ingestion until the first sustained increase of $\mathrm{H}_{2}$ was identified. In the presence of SIBO, the OCTT was estimated as a second peak in $\mathrm{H}_{2}$ concentration values, 60 min after lactulose ingestion.

\section{Procedures}

Patients were randomly assigned to receive the following during a period of 6 months: 1) antibiotics consisting of norfloxacin (Urekolin, Biochemical Beta, Santiago, Chile) (400 mg b.i.d.), and neomycin (Chile Pharmaceutica, Santiago, Chile) (500 $\mathrm{mg}$ t.i.d.) during alternating periods of 15 days; 2) Cisapride (Prepulsid [Janssen Pharmaceutica, Beerse, Belgium]; $10 \mathrm{mg}$ t.i.d.) and 3) placebo (University of Chile, Santiago, Chile) provided in a capsule t.i.d.

Patients had a physical examination and blood pressure, blood count, bilirubin, transaminases, albumin, prothrombin time, urine culture, and creatinine determinations every month. At entry and 3 and 6 months after the beginning of the study, small-intestinal manometry and the $\mathrm{H}_{2}$ breath test with lactulose were performed to investigate SIBO and OCTT. At entry and at 6 months after the beginning of the study, ultrasound was performed to investigate ascites and liver tumors.

\section{Statistics}

Normality was evaluated for each variable. Comparison of variations at 3 and 6 months with baseline for each group of 
Table 1. Characteristics of Cirrhotic Patients at Entry According to Treatment

\begin{tabular}{lccc}
\hline \multicolumn{1}{c}{ Variable } & $\begin{array}{c}\text { Antibiotics } \\
(\mathrm{n}=12)\end{array}$ & $\begin{array}{c}\text { Cisapride } \\
(\mathrm{n}=12)\end{array}$ & $\begin{array}{c}\text { Placebo } \\
(\mathrm{n}=10)\end{array}$ \\
\hline Age, yr (range) & $56(34-68)$ & $58(44-69)$ & $60(49-69)$ \\
Sex (M/F) & $9 / 3$ & $4 / 8$ & $2 / 8$ \\
Alcohol & 9 & 5 & 4 \\
HVC & 2 & 1 & 5 \\
Other etiologies & 1 & 6 & 1 \\
Child-Pugh class A & 0 & 3 & 3 \\
Child-Pugh class B & 7 & 6 & 7 \\
Child-Pugh C & 5 & 3 & 0
\end{tabular}

$\mathrm{HVC}=$ Hepatic C virus infections.

treatment was conducted by means of the paired $t$ test. Comparison among groups at 3 and 6 months was conducted by analysis of covariance, with baseline variable as covariate. The Fisher's exact test was used to evaluated the qualitative characteristic among groups. $p \leq 0.05$ was considered statistically significant.

\section{RESULTS}

Thirty-four patients completed the 6-month period of study. Of those, 12 patients received antibiotics, the same number received cisapride, and 10 received placebo. In this last group, two patients had to be withdrawn because they required antibiotics, one for bacterial peritonitis and the other for urinary tract infection. The characteristics of the three groups are shown in Table 1. Differences in age, etiology, and severity of liver disease observed were not statistically significant. A higher number of women were observed in the placebo group.

\section{Motility Study}

Variation in the different motor parameters analyzed is shown on Table 2. In baseline studies, nonstatistically significant differences in the number of phases III, frequency, and amplitude of contractions, as well as the appearance of clustered contractions were observed among the three groups.

Both groups of patients receiving either antibiotics or cisapride showed a significant improvement in small-intestinal motor activity compared with baseline. Improvement in fasting cyclic activity, defined as the appearance of a phase III when previously absent or as an increase of the number of phases III, if previously present, was observed after 3 months of treatment in six patients given antibiotics, six patients given cisapride, and three patients given pla- cebo. These differences were not statistically significant. However, after 6 months, the improvement of cyclic activity of the MMC was observed in 10 patients given antibiotics, eight given cisapride, and four given placebo; at this time, these differences were statistically significant when comparing both experimental groups with the placebo one. As shown in Table 2, variations of the number of phases III observed after 3 months of treatment were not statistically significant; nevertheless, after 6 months, a statistically significant increase in the number of phases III was observed, from 10 in basal to 30 in patients receiving antibiotics and from 13 in baseline to 26 in patients given cisapride. By contrast, the variation in the number of phases III from 15 to 13 was not statistically significant in patients given placebo. Cyclic activity deteriorated after 6 months in four patients given placebo. Worsening was not observed with antibiotics and cisapride.

No statistically significant variation in the frequency of small-intestinal contractions was observed in the three groups of patients. Amplitude of contractions showed a significant increase in cirrhotics treated with cisapride compared with placebo only after 6 months of treatment. In the antibiotic and placebo groups, there was not a significant variation in the amplitude of contractions.

\section{Orocecal Transit Time}

Table 3 shows mean OCTT. No statistically significant differences in OCTT were observed between the three groups at the basal measurements. No statistically significant variations of OCTT were observed after 3 months. After 6 months, a statistically significant decrease of the OCTT was observed in patients treated both with cisapride or antibiotic. In contrast, OCTT remained unchanged in patients after placebo.

\section{Small-Intestinal Bacterial Overgrowth}

Variations in SIBO in the three groups of treatment are observed in Table 3. No significant differences in the incidence of SIBO were observed among the three groups at basal measurements. The $\mathrm{H}_{2}$ breath test performed after 3 months of treatment showed a nonstatistically significant difference in the number of patients presenting SIBO when comparing cisapride, antibiotic, and placebo with each other. After 6 months of treatment with antibiotics in 10 patients, SIBO was not present, and no additional patient developed SIBO during treatment. A similar finding was

Table 2. Parameters of Small Intestinal Contractile Activity in Patients With Liver Cirrhosis According to Treatment

\begin{tabular}{|c|c|c|c|c|c|c|c|c|c|}
\hline \multirow[b]{2}{*}{ Variable } & \multicolumn{3}{|c|}{ Antibiotics } & \multicolumn{3}{|c|}{ Cisapride } & \multicolumn{3}{|c|}{ Placebo } \\
\hline & Basal & $3 \mathrm{mo}$ & $6 \mathrm{mo}$ & Basal & $3 \mathrm{mo}$ & $6 \mathrm{mo}$ & Basal & $3 \mathrm{mo}$ & $6 \mathrm{mo}$ \\
\hline Phases III (n) & 11 & 20 & $30 * \dagger$ & 13 & 20 & $26 *+$ & 15 & 15 & 13 \\
\hline Frequency (c/min) & $1.18 \pm 03$ & $1.35 \pm 0.3$ & $1.23 \pm 0.4$ & $1.29 \pm 0.5$ & $1.36 \pm 0.4$ & $1.39 \pm 0.5$ & $1.33 \pm 0.5$ & $1.58 \pm 0.5$ & $1.2 \pm 0.5$ \\
\hline Amplitude (mm Hg) & $29.3 \pm 6$ & $26.5 \pm 4.1$ & $26.5 \pm 4.4$ & $28.1 \pm 6.5$ & $32.8 \pm 1.7$ & $30.5 \pm 10^{*}$ & $27.7 \pm 11$ & $26.3 \pm 43$ & $24.1 \pm 4.0$ \\
\hline Clusters (per h) & $8.8 \pm 4.6$ & $6.4 \pm 4.8$ & $5.2 \pm 5$ & $6.8 \pm 5.0$ & $9.1 \pm 7$ & $7.7 \pm 6.0$ & $5.1 \pm 4$ & $7.9 \pm 5.2$ & $4.7 \pm 4$ \\
\hline
\end{tabular}

$* p<0.05$ compared with placebo.

$\dagger p<0.05$ compared with baseline within the group. 
Table 3. Effect of Antibiotic, Cisapride and Placebo on SIBO and OCTT

\begin{tabular}{|c|c|c|c|c|c|c|c|c|c|}
\hline \multirow[b]{2}{*}{ Variable } & \multicolumn{3}{|c|}{ Antibiotics } & \multicolumn{3}{|c|}{ Cisapride } & \multicolumn{3}{|c|}{ Placebo } \\
\hline & Basal & $3 \mathrm{mo}$ & $6 \mathrm{mo}$ & Basal & $3 \mathrm{mo}$ & $6 \mathrm{mo}$ & Basal & $3 \mathrm{mo}$ & $6 \mathrm{mo}$ \\
\hline SIBO (n) & 8 & 6 & $2 *$ & 5 & 3 & $1^{*}$ & 4 & 6 & 6 \\
\hline OCTT (min) & $122 \pm 31$ & $117 \pm 28$ & $97 \pm 38^{*}$ & $113 \pm 26$ & $85 \pm 30$ & $89 \pm 23^{*}$ & $111 \pm 35$ & $111 \pm 34$ & $111 \pm 29$ \\
\hline
\end{tabular}

$* p<0.05$ compared with baseline within the group.

observed with cisapride. By contrast, in the placebo-assigned group, three patients had persistent SIBO, and three additional patients developed SIBO.

To establish which of the different parameters analyzed in the present study were related to the resolution of SIBO, we pooled together all patients without SIBO at the end of the 6-month period and compared this group with those patients with persistent SIBO or with those who developed SIBO during the same period of observation, independent of the therapeutic regimen. Absence of SIBO was significantly related to an increased number of phases III of the MMC and to shortening of OCTT.

\section{Liver Function}

Table 4 shows variations in different parameters of liver function and Child-Pugh status. No significant differences were observed among the three groups in basal estimations. A statistically significant decrease of mean values at 3 and 6 months were observed after a long-term treatment with antibiotics: after 3 and 6 months of treatment, 10 and 11 patients, respectively, showed an improvement in ChildPugh score, and in one patient, the score remained unchanged. A similar finding was observed with cisapride administration, in which an improvement of liver function was seen in eight and 11 patients after 3 and 6 months, respectively. Improvement of Child-Pugh score was mainly related to a statistically significant decrease of the incidence of ascites and encephalopathy compared with basal and placebo levels (Table 4). In the placebo group, mean score variations were not significant, and an improvement of score was observed only in five patients.

\section{DISCUSSION}

The present study shows that a 6-month treatment with both cisapride or antibiotics in patients with liver cirrhosis was followed by 1) an improvement of cyclic activity of the MMC, 2) a significant resolution of SIBO, 3) a significant shortening of OCTT, and 4) an improvement in some parameters of liver function. Cisapride also had additional benefits, such as an increase in the amplitude of smallintestinal contractions.

Our finding on the effect of cisapride on altered smallintestinal motility of patients with cirrhosis is interesting. Despite the fact that motor abnormalities are more frequently seen and are more severe in patients with advanced liver disease, these changes are still reversible with pharmacological treatment. This finding is in accordance with our previous observation of restoration of normal motility after liver transplantation (3) and of altered motility being unrelated to structural changes in the myenteric plexuses (12).

A similar finding on SIBO and OCTT after a short treatment of cisapride in patients with liver cirrhosis and cirrhotic rats was recently reported (13). Six-month treatment with cisapride was followed by a significant resolution of SIBO that was related to the reappearance or improvement of the cyclic activity of the MMC. This is an expected finding because most of the available experimental evidence, including radiation enteritis (9-14) suggests that disturbances of fasting cyclic activity may be followed by SIBO.

The effect of antibiotic treatment is surprising, because neither norfloxacin nor neomycin had effect on GI tract

Table 4. Variation in Different Parameters of Liver Function and Child-Pugh Status

\begin{tabular}{|c|c|c|c|c|c|c|c|}
\hline Group & Mo & Ascitis (n) & Encephalopathy (n) & Bilirubin (mg/dL) & Prothrombin $(\%)$ & Albumin (g/dL) & $\begin{array}{c}\text { Child-Pugh } \\
\text { Score }\end{array}$ \\
\hline \multirow[t]{3}{*}{ Antibotics $(\mathrm{n}=12)$} & 0 & 7 & 5 & $1.8 \pm 1.1$ & $62 \pm 17$ & $3.6 \pm 0.6$ & $9.2 \pm 1.8$ \\
\hline & 3 & 4 & 1 & $1.1 \pm 0.6$ & $63 \pm 15$ & $3.8 \pm 0.6$ & $7.6 \pm 1.8$ \\
\hline & 6 & 1 & 0 & $1.1 \pm 0.6^{*}$ & $69 \pm 17$ & $3.9 \pm 0.6$ & $6.2 \pm 1.2 * \dagger$ \\
\hline \multirow[t]{3}{*}{ Cisapride $(\mathrm{n}=12)$} & 0 & 7 & 4 & $1.5 \pm 0.7$ & $62 \pm 17$ & $3.4 \pm 0.6$ & $8.1 \pm 2.2$ \\
\hline & 3 & 4 & 1 & $1.6 \pm 1.0$ & $60 \pm 15$ & $3.6 \pm 0.6$ & $6.7 \pm 1.6$ \\
\hline & 6 & 3 & 0 & $1.5 \pm 0.9$ & $62 \pm 14$ & $3.8 \pm 0.4^{*}$ & $6.3 \pm 1.6$ \\
\hline \multirow[t]{3}{*}{ Placebo $(\mathrm{n}=10)$} & 0 & 5 & 4 & $1.2 \pm 0.7$ & $63 \pm 8.3$ & $3.4 \pm 1.0$ & $7.7 \pm 1.5$ \\
\hline & 3 & 3 & 3 & $1.4 \pm 0.7$ & $61 \pm 10$ & $3.4 \pm 0.6$ & $6.9 \pm 1.3$ \\
\hline & 6 & 5 & 6 & $1.7 \pm 1.0$ & $60 \pm 8.6$ & $3.2 \pm 0.4$ & $6.6 \pm 1.3$ \\
\hline
\end{tabular}

$* p<0.05$ compared with baseline within group

$\dagger p<0.01$ compared with placebo. 
motility. Changes of small-intestinal motility after antibiotic treatment are very important because the number of phases III recorded on basal conditions increased three times at the end of the study. This result is similar to that observed with cisapride. This effect suggests that SIBO might induce the appearance of alterations of small-intestinal motility. One possible mechanism might be the local production of bacterial endotoxins acting on neurones of the enteric nervous system, which are responsible for the cyclic activity of the small intestine. Experimental evidence suggests that alterations of myoelectrical activity in experimental animals are induced by enterotoxins of different bacteria $(15,16)$ and that in turn, an antiendotoxin effect has been described for neomycin (17). Other studies have shown that neomycin improved OCTT in patients with liver cirrhosis (5). This effect was also observed in our study.

In the present study, disappearance of SIBO was mainly related to an improvement of cyclic activity of small-intestinal motility; these changes were observed with both therapeutic regimens. Shortening of OCTT was another factor associated with the improvement of SIBO.

Recently, Quigley (18) analyzed the possible relationship between altered small-bowel motility, delayed transit time, SIBO, and portal hypertension. Our study have at least in part improved the present knowledge, establishing that SIBO has a role in the appearance of altered motility observed in cirrhotics patients.

Should SIBO be investigated in patients with liver cirrhosis and treated in all of them? Although our results are encouraging, our findings should be confirmed in a series with a greater number of patients.

What of the therapeutic regimens investigated in the present study should be the choice treatment? Cisapride has the advantage that it is an inexpensive drug with additional effects on OCTT and amplitude of contractions. However, whether cisapride is effective in preventing spontaneous bacterial peritonitis, as shown with norfloxacin, was not analyzed in our study. In our selected group of patients, no adverse effects were observed. Cardiovascular effects of cisapride on this group of patients has been previously published; there, neither arrhythmias nor an additional increase in Q-T interval were observed, despite the fact that a mean prolonged basal Q-T was present (19). Interval from the onset of the QRS to the end of T wave is defined as it returns to the $\mathrm{T} / \mathrm{P}$ baseline

In conclusion, long-term treatment with cisapride and antibiotics in liver cirrhosis was followed by an improvement in small-intestinal motility and bacterial overgrowth, and this phenomena might play a role in the management of these patients.

\section{ACKNOWLEDGMENT}

We thank Dr. Luis Carlos Gil La Rotta, who kindly referred his patients to us from San José Hospital. This research was supported by a grant of Fondeayt (W-1970795).
Reprint requests and correspondence: Ana Maria Madrid, M.D., Centro de Gastroenterología Hospital Clínico U. de Chile, Santos Dumont 999-Independencia, Santiago, Chile.

Received Apr. 13, 2000; accepted Aug. 15, 2000.

\section{REFERENCES}

1. Chesta J, Defilippi CL, Defilippi C. Abnormalities in proximal small bowel motility in patients with cirrhosis. Hepatology 1993; 17:828-32.

2. Madrid AM, Cumsille F, Defilippi C. Altered small bowel motility in patients with liver cirrhosis depends on severity of liver disease. Dig Dis Sci 1997;42:738-42.

3. Madrid AM, Brahm J, Buckel E, et al. Orthotopic liver transplantation improves small bowel motility disorders in cirrhotic patients. Am J Gastroenterol 1997;92:1044-5.

4. Chesta J, Lillo R, Defilippi C, et al. Mouth to caecum transit time and solid meal gastric emptying in patients with liver Cirrhosis. Rev Med Chile 1991;119:1248-53.

5. Van Thiel DH, Fagiuoli S, Wright HI, et al. Gastrointestinal transit in cirrhotic patients: Effect of hepatic encephalopathy and its treatment. Hepatology 1994;19:67-71.

6. Martini GA, Phear EA, Ruebner B, et al. The bacterial content of the small intestine in normal and cirrhotic subjects: Relation to methionine toxicity. Clin Sci 1997;16:35-51.

7. Chesta J, Silva M, Thompson L, et al. Small intestine bacterial overgrowth in patients with hepatic cirrhosis. Rev Med Chile 1991:626-32.

8. Casafont F, De Las Heras G, Ramos M, et al. Small bowel bacterial overgrowth in patients with alcoholic cirrhosis. Dig Dis Sci 1995;40:1252-6.

9. Vantrappen C, Janssens J, Hellemans J, et al. The interdigestive motor complex of normal subjects and patients with bacterial overgrowth of the small Intestine. J Clin Invest 1997;59:1158-66.

10. Madrid AM, Defilippi C. Efecto de la cisaprida en la motilidad intestinal de la cirrosis hepática. Gastr Latinoam 1995;6:285.

11. Summers RW, Anuras S, Green I. Jejunal manometry patterns in health, partial intestinal obstruction and pseudo obstruction. Gastroenterology 1983;85:1290-1300.

12. Chesta J, Smok S. Myenteric plexus of the small intestine in patients with liver cirrhosis. Rev Med Chile 1993;121:139-43.

13. Pardo A, Bartolí R, Lorenzo-Zuñiga V, et al. Effect of Cisapride on intestinal bacterial overgrowth and bacterial translocation in cirrhosis. Hepatology 2000;31:858-63.

14. Husebye E, Viggo S, Hovertad T, et al. Abnormal intestinal motor patterns explain enteric colonization with gram-negative in late radiation enteropathy. Gastroenterology 1995;109:1078-89.

15. Justus P, Martin J, Golberg D, et al. Myoelectric effects of clostridium difficile: Motility-altering factors distinct from its cytotoxin and enterotoxins in rabbits. Gastroenterology 1982;83:836-45.

16. Sninsky C, Ramphal R, Gaskins D, et al. Alterations of myoelectric activity associated with campylobacter jejuni and its cell-free filtrate in the small intestine of rabbits. Gastroenterology 1985;89:337-44.

17. Nolan JP. Intestinal endotoxins as mediators of hepatic injury. An idea whose time has come again. Hepatology 1989;10:887-91.

18. Quigley E. Gastrointestinal dysfunction in liver disease and portal hypertension. Gut-liver interaction revisited. Dig Dis Sci 1996;41:557-63.

19. Quera R, Madrid AM, Ugalde H, et al. Cisapride does not modify prolonged Q-T interval in patients with liver cirrhosis. Rev Med Chile 2000;128:847-52. 